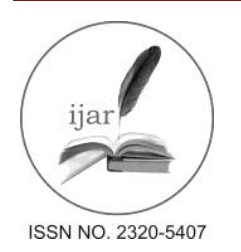

Journal homepage: http://www.journalijar.com

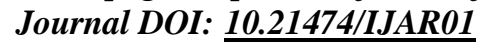

RESEARCH ARTICLE

\title{
PREVALENCE AND EFFECT OF SEN VIRUS IN HEMODIALYSIS PATIENTS IN ZAGAZIG UNIVERSITY NEPHROLOGY UNIT.
}

\author{
Said M. Al-Barshomy, ${ }^{1}$ Nafesa M. Kamal, ${ }^{1}$ Ahmed Abo-Hassan ${ }^{2}$ and Awny Ali Gawish ${ }^{3}$ \\ 1. Internal medicine department - Zagazig University -Egypt. \\ 2. Internal medicine department - Al-Azhar University -Egypt. \\ 3. Microbiology and virology department- Zagazig University- Egypt.
}

\section{Manuscript Info}

Manuscript History:

Received: 12 April 2016

Final Accepted: 16 May 2016

Published Online: June 2016

Key words:

SEN virus, hemodialysis, prevalence, Zagazig

*Corresponding Author

Said M. Al-Barshomy.

\section{Abstract}

Background: Chronic hemodialysis patients are at higher risk of multiple types of infections than other normal population, especially blood-borne. About $5 \%$ of those patients had a full picture of acute hepatitis without known causative agent for this unknown none A-E hepatitis e.g. TTV, GBV$\mathrm{C}$ and SEN virus (SENV), but after thorough investigations, these viruses were discounted as causes of serious hepatitis.

SENV is a single-stranded, non-envelped, circular DNA which was considered as a member of circoviridae family, but it has been recently classified in a floating genus named Anellovirus: unclassified Anellovirus which also include Anellovirus PRA1 (felis catus anellovirus PRAI), Anellovirus PRA4 (felis catus anellovirus PRA 4), SEN-V, Small Anellovirus, TTV (transfusion transmitted virus), TTMV (torque teno mini virus) and TTMDV (torque teno midi virus).

SENV has nine different genotypes (A to I) with at least $25 \%$ divergence in nucleotide sequence. SENV-D and SENV-H genotypes have comparatively higher frequencies in post- transfusion non A-E hepatitis. Additionally SENV-H has been found more frequent in hemodialysis patients. The main route of transmission is parentral via transfusion of blood and its products, however, other routes have been documented such as feco-oral, sexual and vertical transmissions. It can be detected by PCR and has no treatment.

Aim of the work: The aim of this study is to screen high risk group i.e. chronic hemodialysis patients and to identify any ability of SENV to induce acute hepatitis or aggravating the already existing chronic hepatitis.

Patients and methods: The study included 98 ESRD patients on regular HD screened for SENV and divided in to 2 groups, SENV +ve and SENV -ve groups.

The 98 patients were subjected to proper history taking including name, age, sex, occupation, past history of blood transfusion, liver disease and duration of hemodialysis was obtained. Full clinical assessment for relevant clinical signs. Screening for anti-HCV as a marker for $\mathrm{HCV}$ and for $\mathrm{HBsAg}$ as a marker for $\mathrm{HBV}$ as a confirmation for previous virology results recorded in patients' files, liver function tests and complete blood picture. Screening for SENV-DNA by polymerase chain reaction (PCR) and liver ultrasound. 
Results: prevalence of SENV infection among patients was only $4.1 \%$. In our study, there were no association between the positivity rates of SENV and the demographic data (age-gender- living area), history of blood transfusion, history of previous surgeries, history of co-morbidities (hypertension, diabetes or both), sera status for anti-HCV antibodies and/or HBs Ag or history of past surgery.

SENV alone failed to cause any liver insult in those without any liver illness before and negative for anti-HCV and/or HBsAg. Additionally, it failed to exacerbate or initiate liver insult in our patients with positive sera for anti$\mathrm{HCV}$ antibodies.

The effect of SENV on hematopoiesis in our patients has not been clearly established and there has not been any statistically significant effect on WBC, hemoglobin and platelets count.

Conclusion: SEN V infection is uncommon (only 4.1\%) among HD patients in Zagazig university hospitals nephrology unit. There is no association between age, gender, area of living, history of blood or its products transfusion, history of past surgeries, $\mathrm{HCV}$ and/or HBsAg and history of common co-morbidities in ESRD e.g. diabetes and/or hypertension, and the presence of SENV in the sera of chronic hemodialysis patients. SENV has no relation to acute liver insult or to aggravation of pre-existing liver illness.

Copy Right,IJAR, 2016. All rights reserved.

\section{Introduction:-}

Infection is the most common cause of hospitalization and the second most common cause of mortality among hemodialysis patients. The increased risk for infections including blood-borne pathogens in such patients is mainly due to (a) immune compromised status, (b) frequent and prolonged blood exposure during hemodialysis treatments through the vascular access and extra-corporeal circuit, c) close proximity to other patients during treatment in the hemodialysis unit, (d) frequent contact with health care workers who frequently move between patients and between machines (e) frequent blood-products transfusions, hospitalization and surgery and most importantly (f) nonadherence or a break in implementation of recommended practices for infection control. ${ }^{1}$

Several lines of evidence have suggested the existence of new hepatitis agents, in addition to established hepatitis viruses A-E. Before I990, 10-20\% of patients with both transfusion- associated and community-acquired hepatitis tested were negative for known hepatitis viruses. Recently, a new virus of single- stranded DNA with hepatotropic properties was isolated and designed as SEN virus (SENV) by an Italian research group. ${ }^{2}$

There are 9 genotypes of SENV designed as A-I, the most frequent are D and $\mathrm{H}$ which are the most closely associated with transfusion-associated non A-E hepatitis. ${ }^{3}$ Moreover, it was observed that prevalence of SENV-H was more than SENV-D in hemodialysis patients and even in control healthy group. ${ }^{4}$

SEN-V has parenteral mode of transmission, However; non parentral modes of transmission such as feco-oral, sexual and vertical transmissions have been also reported. ${ }^{5}$

Although SENV was initially considered a possible agent of non A-E hepatitis, after thorough clinical investigation it was discounted as 21 causal agent of significant liver disease. Moreover, SENV has been thought recently to be a simple guest causing mild self-limited acute hepatitis (if any) without any chronic hepatitis or cirrhosis, in addition it failed to aggravate the already existing liver disease. ${ }^{6}$ 


\section{Aim of the work:-}

Comparative cross-sectional study to determine the prevalence and effect of SEN-V in high risk subjects as patients on hemodialysis in the dialysis unit of the internal medicine department Zagazig University

\section{Subjects and method:-}

This study has been performed in the period from Dec 2014 to Sep. 2015 in internal medicine and nephrology unit Zagazig University hospitals.

After the exclusion of eight patients according to specific criteria, this comparative cross-sectional study has included 98 patients of ESRD on long-term hemodialysis after their agreement to screen the prevalence of SENV among them and to study its possible effects.

\section{Inclusion criteria:-}

This study has aimed to include all patients, who are known to have End stage renal disease on long-term hemodialysis in the dialysis unit of internal medicine department in Zagazig University as long as they agree and do not have any of the exclusion criteria.

Patients have different ages, genders, occupations, past-history of blood transfusions, past-history of previous surgeries and durations on hemodialysis. They live in Zagazig city or in the rural areas around it. Patients also have different causes of End stage renal disease and have different co-morbidities e.g. diabetes, hypertension or both. Patients have different patterns of their sera, which is either positive for anti-HCV and/or HBsAg or negative for both. Those who have positive sera for anti-HCV, HBsAg or both may have liver disease.

\section{Exclusion criteria:-}

Patients with severe liver disease, malignancy either hepatocellular carcinoma or extra-hepatic malignancies and patients under immuno-suppressive therapy was excluded according to previous complete blood picture results recorded in patients' files those who were suspected to have aplastic anemia. The idea to exclude such patients was to avoid any undesirable influences on our results.

\section{Patients selection and classification:-}

After detection of positive patients for SENV by polymerase chain reaction (PCR), the 98 patients have been classified into SENV-positive and SENV-negative groups and they were both compared according to data obtained by history, from patients' files and serology results, i.e. age, gender, area of living, duration on hemodialysis in years, average hours of hemodialysis session per week (hours/week), history of blood transfusion or its products, history of previous surgeries, history of co-morbidities e.g. hypertension and/or diabetes and the result of serology whether they are positive or negative for anti-HCV and/ or HBsAg.

To study the effect of SENV, each group of aforementioned two groups i.e. SENV-positive and -SENV-negative have been divided into groups according to their serology:

1. SENV-positive with negative sera for both anti-HCV and HBsAg.

2. SENV-positive with positive sera for anti-HCV alone.

3. SENV-positive with positive sera for HBsAg alone.

4. SENV-negative with negative sera for both anti-HCV and HBsAg "uninfected": the control group for SENVpositive with negative sera for both anti-HCV and HBsAg.

5. SENV-negative with positive sera for anti-HCV alone: the control group for SENV-positive with positive sera for anti- HCV.

6. SENV-negative with positive sera for HBsAg alone: the control group for SENV-positive with positive sera for HBsAg alone.

There were no patients whose sera were positive for HBsAg were positive for SENV, so both groups number 3 and number 6 were excluded at this step. Additionally, the only patient who had HBsAg and anti-HCV was SENVnegative and he hasn't been added to any group. 
The groups 1, 2, 4 and 5 were compared, each to its controller as mentioned before regarding components of complete blood picture, liver function tests and ultrasound imaging of the liver which was done for patients mainly to detect liver size in long axis, to exclude any focal lesion in the liver, to detect the patency and caliber of the portal vein, to rule out fatty infiltration and to examine the gall bladder for its wall thickness or any stone.

\section{Methods:}

The 98 patients were all candidate for the following after written agreement signed by them: Proper history taking including name, age, sex, occupation, past history of blood transfusion, liver disease and duration of hemodialysis was obtained. Full clinical assessment for relevant clinical signs. Screening for anti-HCV as a marker for HCV and for HBsAg as a marker for HBV as a confirmation for previous virology results recorded in patients' files, Liver function tests and complete blood picture.

Screening for SENV-DNA by polymerase chain reaction (PCR) and liver ultrasound.

Specimen collection and preparation:

$5 \mathrm{ml}$ of blood was collected in a clean test tube without any anticoagulant using aseptic technique. Each specimen was spun down, within one hour of its collection, at 3000 round per minute (r.p.1n) for 10 minutes. The serum was divided into two aliquots, one of them was sent to laboratory for liver function tests and the other was stored at $-20^{\circ}$ till it was tested for serology (anti-HCV and HBsAg) and detection of SENV-DNA by PCR. Another $2 \mathrm{ml}$ of blood was collected in a purple tube containing EDTA as an anticoagulant and was sent to the lab within 24 hours to be examined for complete blood count.

Detection of SEN- $\mathrm{V}$ by polymerase chain reaction $(\mathrm{PCR})$ :-

All patients were screened for SEN-V using polymerase chain reaction (PCR).

\section{Extraction of viral DNA:}

Viral DNA was extracted from 200 ul serum with QlAamp DNA blood mini kit (Qiagen, Cat. No.51104-Germany).

\section{Analytical statistics:}

Data were fed to the computer and analyzed using IBM SPSS software package version 20.0. ${ }^{7}$ Qualitative data were described using number and percent. Quantitative data were described using range (minimum and maximum), mean, standard deviation and median. Significance of the obtained results was judged at the $5 \%$ level.

\section{Results:}

Table 1:- prevalence of SENV in HD patients.

\begin{tabular}{|c|c|c|}
\hline SEN virus & No. & $\%$ \\
\hline Negative & 94 & 95.9 \\
\hline Positive & 4 & 4.1 \\
\hline
\end{tabular}

\begin{tabular}{|c|c|c|c|c|c|c|}
\hline \multirow{3}{*}{ Gender } & \multicolumn{4}{|c|}{ SEN virus } & & \multirow{2}{*}{$\begin{array}{l}\text { Total } \\
\mathrm{N}=98\end{array}$} \\
\hline & \multicolumn{2}{|c|}{$\begin{array}{c}\text { Negative } \\
\mathrm{N}=94\end{array}$} & \multicolumn{2}{|c|}{$\begin{array}{c}\text { Positive } \\
\mathrm{No}=4\end{array}$} & & \\
\hline & $\mathrm{No}$ & $\%$ & No & $\%$ & $\mathrm{No}$ & $\%$ \\
\hline Male & 53 & 56.4 & 3 & 75 & 56 & 57.1 \\
\hline Female & 41 & 43.6 & 1 & 25 & 42 & 42.9 \\
\hline $\left.\mathrm{X}^{2(\mathrm{FE}} \mathrm{p}\right)$ & & & & & & \\
\hline & & & r $\mathrm{Eq}$ & & & \\
\hline
\end{tabular}




\begin{tabular}{|c|c|c|c|}
\hline Table 3:- demographic data of the 2 studied groups according to age. & \multirow{2}{*}{$\begin{array}{c}\text { Total } \\
\text { Nge (years ) }\end{array}$} \\
\cline { 2 - 4 } & $\begin{array}{c}\text { Negative } \\
\text { N=94 }\end{array}$ & $\begin{array}{c}\text { Positive } \\
\text { No=4 }\end{array}$ \\
\cline { 2 - 4 } & $20.0-77.0$ & $22.0-55.0$ & $20.0-77.0$ \\
\hline Min - Max & $46.23 \pm 14.34$ & $42.50 \pm 14.39$ & $46.08 \pm 14.28$ \\
\hline Mean \pm SD & 49.50 & 46.50 & 49.0 \\
\hline Median & \multicolumn{3}{|c|}{$0.510(0.611)$} \\
\hline T $(\mathrm{P})$ & \multicolumn{2}{|c|}{ T student t- test } \\
\hline
\end{tabular}

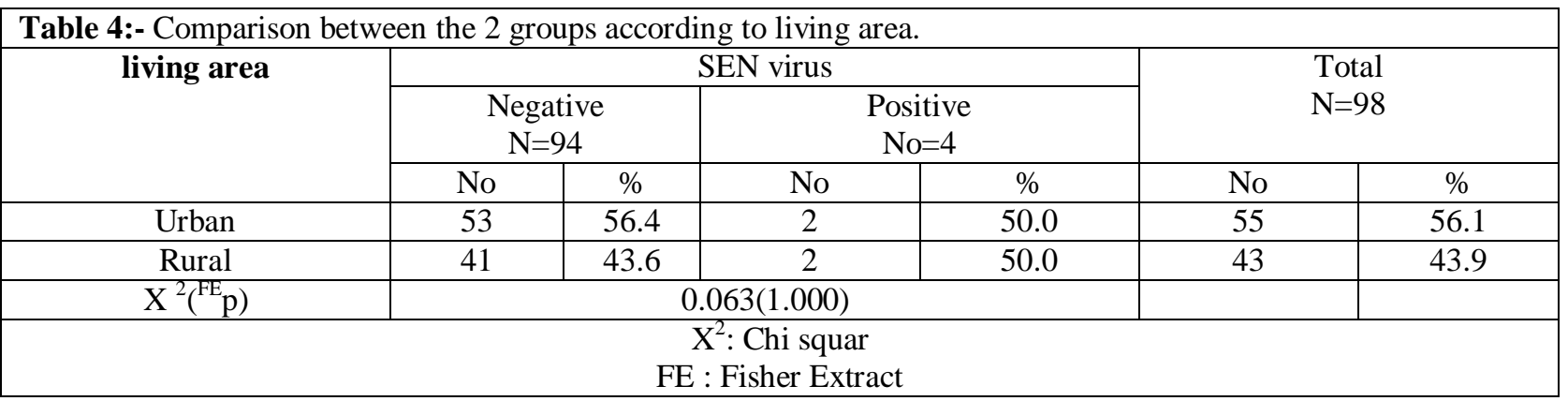

\begin{tabular}{|c|c|c|c|c|c|c|}
\hline \multirow{3}{*}{$\begin{array}{l}\text { history of blood or its } \\
\text { products transfusion }\end{array}$} & \multicolumn{4}{|c|}{ SEN virus } & \multirow{2}{*}{\multicolumn{2}{|c|}{$\begin{array}{l}\text { Total } \\
\mathrm{N}=98\end{array}$}} \\
\hline & \multicolumn{2}{|c|}{$\begin{array}{c}\text { Negative } \\
\mathrm{N}=94\end{array}$} & \multicolumn{2}{|c|}{$\begin{array}{c}\text { Positive } \\
\text { No=4 }\end{array}$} & & \\
\hline & No & $\%$ & No & $\%$ & No & $\%$ \\
\hline No & 10 & 10.6 & 0 & 0.0 & 10 & 10.2 \\
\hline Yes less than 5 units & 29 & 30.9 & 0 & 0.0 & 29 & 29.6 \\
\hline Yes more than 5 units & 55 & 58.5 & 4 & 100.0 & 59 & 60.2 \\
\hline $\mathrm{X}^{2}\left({ }^{\mathrm{mc}} \mathrm{p}\right)$ & \multicolumn{4}{|c|}{$1.786(0.410)$} & & \\
\hline \multicolumn{7}{|c|}{$\begin{array}{c}\mathrm{X}^{2}: \text { Chi squar } \\
\text { Mc : monte carlo }\end{array}$} \\
\hline
\end{tabular}

\begin{tabular}{|c|c|c|c|c|c|c|}
\hline \multirow[t]{3}{*}{ Virology } & \multicolumn{4}{|c|}{ SEN virus } & & \\
\hline & \multicolumn{2}{|c|}{$\begin{array}{c}\text { Negative } \\
\mathrm{N}=94\end{array}$} & \multicolumn{2}{|c|}{$\begin{array}{c}\text { Positive } \\
\mathrm{No}=4\end{array}$} & \multicolumn{2}{|c|}{$\begin{array}{l}\text { Total } \\
\mathrm{N}=98\end{array}$} \\
\hline & No & $\%$ & No & $\%$ & No & $\%$ \\
\hline -ve HBsAg-ve and anti HCV & 43 & 458 & 1 & 25.0 & 44 & 44.9 \\
\hline +ve HBsAg & 7 & 7.4 & 0 & 0.0 & 7 & 7.1 \\
\hline +ve anti HCV & 43 & 45.8 & 3 & 75.0 & 46 & 47.0 \\
\hline +ve HBsAg-ve and anti HCV & 1 & 1.0 & 0 & 0.0 & 1 & 1.0 \\
\hline $\mathrm{X}^{2}\left({ }^{\mathrm{mc}} \mathrm{p}\right)$ & \multicolumn{4}{|c|}{$2.525(0.731)$} & & \\
\hline \multicolumn{7}{|c|}{$\mathrm{X}^{2}$ : Chi squar } \\
\hline
\end{tabular}




\begin{tabular}{|c|c|c|c|}
\hline Table 7:- platelet count in SEN +ve and -ve groups. & Total \\
\hline Platelet count & SEN +ve & SEN -ve & N=44 \\
$150-450$ & $\mathrm{HCV}-\mathrm{ve}$ & $\mathrm{HCV}-\mathrm{ve}$ & \\
& $\mathrm{HBsAg}-\mathrm{ve}$ & $\mathrm{HBs}-\mathrm{Ne}$ & \\
& $\mathrm{N}=1$ & $74.0-305.0$ & $74.0-305.0$ \\
\hline Min- Max & - & $195.47 \pm 55.71$ & $195.56 \pm 50.07$ \\
\hline Mean \pm SD & 200 & 191.00 & 191.5 \\
\hline Median & - & & \\
\hline $\mathrm{T}(\mathrm{p})$ & \multicolumn{2}{|c|}{$0.080(0.936)$} \\
\hline
\end{tabular}

The prevalence of SENV infection in our patients is $4.1 \%$, (table 1).

No significant difference between groups (SENV +ve and SENV-ve) as regards age, gender, duration of dialysis, past surgery and living area, table 2and 3 and 4 .

No significant difference between groups (SENV +ve and SENV-ve) as regards history of blood transfusion or even number of units received, (Table 5).

No significant difference between groups (SENV + ve and SENV-ve) as regards history of aforementioned comorbidities.

In SENV +ve group : 3 out of 4 patients in this group (75\%) are anti HCV +ve while only 1 patient (25\%) who is ve for both $\mathrm{HCV}$ and HBsAg and there is none of SENV +ve patients has HBsAg alone or in combination with anti $\mathrm{HCV}($ table 6$)$..

In SENV -ve group : 43 patients in this group (45.8\%) are negative for both anti $\mathrm{HCV}$ and $\mathrm{HBsAg}, 7$ patients $(7.4 \%)$ are +ve for HBsAg , 43 patients in this group (45.8\%) are +ve for HCV antibody and only 1 patient is +ve for both $\mathrm{HCV}$ and HBsAg (table 6).

there was no significant statistical difference between the SENV-positive and -negative patients in relation to liver size in long axis, regardless to positivity or negativity of anti- HCV, so liver ultrasound images did not seem to show any big difference between SENV-negative and SENV-positive patients

There was no significant difference between the 2 groups as regards serology status of HCV and HBsAg. Also no significant difference as regards wbc, hemoglobin and platelet count (table 7).

\section{Discussion:-}

Only 4 patients of 98 patients (4.1\%) were positive for SENV by PCR using specific primers reported by Umemura et al., (2001). ${ }^{8}$ This result shows that such infection seems to be uncommon among chronic hemodialysis patients who undergo dialysis in Zagazig university hospitals, in accordance with Omar et al., (2008) ${ }^{9}$ who reported that SENV did not seem to be a common infection in Egyptian patients and the prevalence of SENV was $13.5 \%$ among patients of chronic liver disease not on hemodialysis, $11.1 \%$ among patients of chronic liver disease on hemodialysis and $7.1 \%$ among healthy controllers. On the other hand, EIKeraie et al., (2015) ${ }^{\mathbf{1 0}}$ reported a relatively high (59\%) prevalence of SENV among chronic hemodialysis patients in Alexandria university hospitals. Reports from Italy, USA, Germany and Japan have documented a range of infection from $12.8 \%$ to $38 \%$ in chronic hemodialysis patients. The reasons for these demographic differences are unclear, but we agreed with Spataro et al., (2006) ${ }^{11}$, who mentioned that SENV prevalence had been variable from one unit to another even within the same country may be due to the difference in the quantity of SENV DNA in the sera, the difference in PCR primers used, the difference in sample storage time or the difference in the sensitivity to the assay used. Additionally, the difference in the rate of prevalence of an infectious agent in hemodialysis units may be due to a variation of the universal precautions and standard infection control measures including transfusion practices and hygienic standards which could be found among different hemodialysis units even within the same country or geographical region. 
In our study, there was no statistical difference in the positivity rate of SENV in relation to age in accordance with Abd El-Hadi et al., (2006) ${ }^{12}$, Yoshida et al., (2001) ${ }^{13}$ and Eldin and Ghandour, (2011) ${ }^{14}$. On the other hand, ElKeraie et al., (2015) ${ }^{10}$ reported that the SENV was more prevalent in older patients on long-term hemodialysis. Although $75 \%$ of SENV-positive patients in our present study were males, there was no statistical significant difference of SENV prevalence in relation to gender between SENV-positive and -negative patients, the same which Yoshida et al., (2001) ${ }^{13}$, Pirovano et al., $(\mathbf{2 0 0 5})^{15}$, ElKeraie et al., $(\mathbf{2 0 1 5})^{10}$, Loutfy et al., (2009) ${ }^{3}$ and Omar et al., (2008) $)^{9}$.

On the other hand, Kobayashi et al., (2003) ${ }^{16}$, Spataro et al., (2006) ${ }^{11}$ and Schreter et al., $(\mathbf{2 0 0 6})^{17}$ reported a notable difference in SENV prevalence according to gender in chronic hemodialysis patients and they claimed that male patients on hemodialysis tended to retain SENV viremia longer than female patients.

In this study, there was no statistical significant difference in SENV positivity rates in relation to area of living whether urban or rural, in accordance with ElKeraie et al., (2015) ${ }^{10}$, but more extended surveys may be needed to verify if the type of area can affect the SENV positivity or not. In our study, there was no relationship between SENV positivity and the duration on hemodialysis, in harmony with Eldin and Glzandour, (2011) $)^{14}$, Omar et al., $\mathbf{( 2 0 0 8}^{9}$, Abd El-Hadi et al., (2006) ${ }^{12}$, Loutfy et al., (2009) ${ }^{3}$ and Dai et al., $(\mathbf{2 0 0 5})^{18}$. On the other hand, ElKeraie et al., (2015) ${ }^{10}$ noticed that higher SENV positivity rates were found in patients with longer duration of hemodialysis this study, although $100 \%$ of SENV-positive patients had positive history for blood transfusions, there was no statistical significant difference in SENV positivity rates in relation to history of previous blood transfusion and no association between the rate of SENV positivity and the number of units of blood transfused, in the harmony with Yoshida et al., (2002) and Omar et al., (2008) ${ }^{9}$. On the other hand, ElKeraie et al., (2015) ${ }^{10}$, Abd El-Hadi et al., (2006) ${ }^{12}$, Umemura et al., (2001) ${ }^{8}$ and Shibata et al., (2001) ${ }^{19}$ mentioned that there were high significant positivity rates of SENV in patients with history of blood transfusion.

Our patients have not reported a history for any major complicated surgeries, but most of them gave a history of only minor surgeries e.g. appendectomy and herniorrhaphy, which did not require blood transfusion or long hospital stay. Additionally, it seems like other non-parentral routes e. g. feco-oral has a role among our patients, in the harmony with Omar et al., (2008). ${ }^{9}$

In our study the relation between SENV prevalence and co-morbidities like diabetes and hypertension has been investigated. $50 \%$ of SENV- positive patients are hypertensive and no one of them had diabetes alone, but there was $25 \%$ of them had both diabetes and hypertension. There was no statistical significant difference in SENV positivity rates in relation to these co-morbidities. There are few data about whether the prevalence of SENV is affected by such co-morbidities or no, so a lot of further studies should be done to study such relation (if any). In the present study, there was no significant statistical difference between SENV-positive patients and SENV-negative patients in relation to the presence of absence of anti-HCV and/or HBsAg, in accordance with Omar et al., (2008), ${ }^{9}$ who reported that there were no statistically significant differences for the distribution of HCV or hepatitis B virus (wellknown blood- borne viruses) between SEN virus-infected and non-infected hemodialysis patients. A possible explanation is that SEN virus can be transmitted through not only parenteral but also non-parenteral routes. '

In our study, 75\% SENV-positive patients were anti-HCV positive, in the accordance with ElKeraie et al., (2015) ${ }^{10}$, who reported SENV and HCV in 55.7\% of anti-HCV positive patients and Abd El-Hadi et al., (2006), who reported SENV in $66.7 \%$ of anti-HCV positive patients. On the other hand, lower rates of SENV in anti-HCV positive patients were reported by Bowden et al., (2001) ${ }^{20}$, Sagir et al., (2004) ${ }^{21}$ and Kobayashi et al., (2003) ${ }^{16}$, who reported rates of $20 \%, 22 \%$ and $19 \%$, respectively. The observed frequency of HCV and SENV is a logic thing because both of them can be transmitted parentrally via blood transfusion or via the iatrogenic means in the dialysis unit setting e. g. multi-dose vial of drugs.

In this study, none of SENV-positive patients had HBsAg. In contrary to many reports which have documented variable rates of SENV infection among HBsAg-positive patients. Abd El-Hadi et al., (2006), Kao et al., (2002), Bowden et al., (2001), and XU et al., (2004) ${ }^{22}$.In our study, the absence of SENV in HBsAg-positive patients enhances our conclusion about non-parentral route of SENV transmission and it may be also because the number of HBsAg-positive patients is limited in our unit (only 7 patients). 
In our study, all the 4 SENV-positive patients had normal values of serum aminotransferases, serum albumin, and total bilirubin. In our study, SENV failed to induce hepatitis in the only SENV-positive patient with negative sera for both anti-HCV and HBsAg and it did not cause any significant difference in LFT, compared to SENV-negative patients with negative sera for both anti-HCV and HBsAg, in accordance with Shibata et al., (2001), Eldin and Ghandour, (2011), Kobayashi et al., (2003), Abd El-Hadi et al., (2006), Sagir et al., (2004) and Schreter et al., (2006). On contrary to our results, Umemura et al., (2001) reported elevated liver enzymes in SENV-positive patients.

there was no significant statistical difference between the SENV-positive and -negative patients in relation to liver size in long axis, regardless to positivity or negativity of anti- HCV, so liver ultrasound images did not seem to show any big difference between SENV-negative and SENV-positive patients, in accordance with Pirovano et al., (2005). ${ }^{15}$

In our study, platelet count in the patient who is SENV- positive with negative sera for both anti-HCV and HBsAg is a little bit higher than the mean of the platelet counts for the 43 patients who are SENV-negative with negative sera for both anti-HCV and HBsAg "uninfected", but this was not statistically significant. Moreover, there was no significant statistical difference between the mean WBCs count in patients who are SENV-positive with positive sera for anti-HCV and that for the 43 patients who are SENV-negative with positive sera for anti-HCV. Also there was no statistical significance between the two groups regarding to hemoglobin). In contrary to ElKeraie et al., (2015). ${ }^{10}$ Anemia can be explained in such patients as a cause of chronic illness.

\section{Conclusion:-}

SEN V infection is relatively uncommon infection (only 4.1\%) among HD patients in the dialysis unit of Zagazig university hospitals. There is no association between age, gender, area of living, history of blood or its products, history of past surgeries, HCV and/or HBsAg and history of common co-morbidities in ESRD e.g. diabetes and/or hypertension, and the presence of SENV in the sera of chronic hemodialysis patients. SENV failed to induce acute liver insult or to aggravate pre-existing liver illness.

\section{References:-}

1. Karkar A, Bouhaha BM, and Dammang ML (2014): Infection control in hemodialysis units: A quick access to essential elements. Saudi Journal of Kidney Diseases and Transplantation. May 1;25(3):496.

2. Allain JP. (1999): Emerging viruses in blood transfusion. Vox sanguinis. Dec; 783243-8.

3. Loutfy SA, Hafez MM, Masoud WA, et al., (2009): SEN-V infection in Egyptian patients undergoing maintenance hemodialysis prevalence and clinical importance. J Microbiol, lmmunol and infect.;42(6); 464470.

4. Kao JH, Chen W, Chen PJ, et al., (2003): SEN virus infection in patients with chronic hepatitis C: preferential coinfection with hepatitis $\mathrm{C}$ genotype $2 \mathrm{a}$ and no effect on response to therapy with interferon plus ribavirin. Journal of Infectious Diseases. Jan 15;187(2):307-10.

5. Borawski J, Kovalchuk O, Chlebinska I, et al., (2006): SEN virus infection in maintenance Hemodialysis patients. KlinikiNefrologiTransplantologii . 9(11-12):751-756.

6. Husseini-Moghaddam SM, Mousavi SH and Alavian SM (2009): Is SEN virus a major concern in hemodialysis and liver transplantation. Hepai Mon. Jan 1;9(1):50-9 .

7. Kirkpatrick LA and Feeney BC. (2013): A simple guide to IBM SPSS statistics for version 20 Student ed. Belmont, Calif: Wadsworth, Cengage Learning.

8. Umemura T, Yeo AE, Sottini A, et al., (2001): SEN virus infection and its relationship to transfusionassociated hepatitis. Hepatology. May 31;33(5):1303-11.

9. Omar M, El-Din SS, Fam N, et al., (2008): SEN virus infection in Egyptian patients with chronic hepatitis C and patients undergoing hemodialysis. The Medscape Journal of Medicine.;10(12):290.

10. Elkeraie AF, Elbably E, Selim H and Hafez E.(2015): SEN virus among Egyptian hemodialysis patients and blood donnors. Nephrology Dialysis \& Transplantation. May 1;30(3): 618 -9.

11. Spataro P, Di Pietro A, Elena Scoglio M, et al., (2006): Prevalence of SENV-H and SENV-D virus: epidemiological study in blood donors and dialysis patients. Renal failure. 2006;28(5):441-8.

12. Abd El Hady SI, Zaki SM, El-Sayed AA, et al., (2006): Prevalence of SENV infection among patients on maintenance hemodialysis in Egypt. Egy .1 Med Microbial. Jan;15(1): 99-112. 
13. Yoshida EM, Buczkowski AK, Giulivi A, et al., (2001): A cross-sectional study of SEN virus in liver transplant recipients. Liver transplantation. Jun 1;7(6):521-5.

14. Eldin SS and Ghandour AM. (2011): Prevalence of SEN Virus Infection in Multitransfused Patients in Assiut University Hospitals, Egypt. Journal of A merican Science. 7(1).

15. Pirovano S, Gregorini G, Malacarne F, et al.,( 2005): High prevalence of SEN virus infection in patients on maintenance hemodialysis: frequent mixed infections with different variants and evidence for nosocomial transmission of the virus. lntervirology.;48 (4) $1216-22$.

16. Kobayashi N, Tanaka E, Umemura T, et al., (2003): Clinical significance of SEN virus infection in patients on maintenance hemodialysis. Nephrol Dial Transplant. Feb; 18(2); 348-52.

17. Schreter I, Kristian P, Jarcuska P et al., (2006): A detection of SEN virus in the general population and different risk groups in slovenia.folia microbiologica. May 1;51(3):223-8).

18. Dai CY, Chuang WL, Chang WY, et al., (2005): SEN virus infection among patients on maintenance hemodialysis in southern Taiwan. Journal of Infection. Aug 31;51(2):1 10-5.

19. Shibata M, Wang RY, Yoshiba M, et al., (2001): The presence of a newly identified infectious agent (SEN virus) in patients with liver diseases and in blood donors in Japan. Journal of infectious diseases. Aug $15 ; 184(4): 400-4$.

20. Bowden S, (2001): new hepatitis; considers and pretenders. Journal of gastroenterology and hepatology. Feb 1;16(2):124-31.

21. Sagir A, Kirschberg O, Heintges T et al., (2004): SEN virus infection. Rev Med Viral. May-Jun;14(3): 141-8.

22. XUD, Tian DY, Zhang ZG et al., (2004): effect of SEN virus co - infection on outcome of lamivudine therapy in patients with hepatitis B. world journal of gastroenterology. Apr 1;10 (7):968-71. 